\title{
Nonlinear Optical Rectification and Second Harmonic Generation in 2D Quantum Rings under Electric Field and Magnetic Fields
}

\author{
A.L. Morales ${ }^{a}$, M.E. Mora-RAmos ${ }^{a, b, c}$ And C.A. DuquE ${ }^{a}$ \\ ${ }^{a}$ Grupo de Materia Condensada-UdeA, Instituto de Física, Facultad de Ciencias Exactas y Naturales \\ Universidad de Antioquia UdeA, Calle 70 No. 52-21, Medellín, Colombia \\ ${ }^{b}$ Facultad de Ciencias, Universidad Autónoma del Estado de Morelos, Cuernavaca, México \\ ${ }^{c}$ Física Teórica y Aplicada, Escuela de Ingeniería de Antioquia, Envigado, Antioquia, Colombia
}

\begin{abstract}
By using the effective mass and parabolic band approximations, the nonlinear optical rectification and the second harmonic generation are calculated in a two-dimensional quantum ring under the simultaneous effects of perpendicularly applied magnetic field and in-plane applied electric field. The geometry of the ring is modeled via the combined influences of a parabolic and inverse square confining potentials. The exact solutions for the two-dimensional motion of the conduction band electrons are used as the basis for a perturbation-theory treatment of the static electric field effect. The variation of one of the different potential energy parameters, for a fixed configuration of the remaining ones, leads to either blueshifts or redshifts of the resonant peaks as well as to distinct rates of change for their amplitudes.
\end{abstract}

DOI: 10.12693 /APhysPolA.125.195

PACS: 42.65.-k, 73.21.-b, 78.67.-n

\section{Introduction}

Incorporating externally applied electric and magnetic fields may result in significant modifications of the electron energy spectrum in quantum heterostructures. This certainly affects a large number of properties in them. One of the most important repercussions is the possibility of obtaining strong nonlinear optical responses, resulting from the consequent increment in the expected values of the electron dipole moment. Among these properties, it is of interest the study of second-order coefficients such as the nonlinear optical rectification (NOR) and the second harmonic generation (SHG) ones [1].

Recent modeling of quantum discs plus parabolic and inverse square potentials has led to a plausible analytical description that can be used to obtain the spectrum of electron states in two-dimensional quantum rings (QRs) under the influence of externally applied magnetic fields $[2,3]$. In this work we are going to use the advantages of this model approach to study the NOR and SHG in 2D QRs in the presence of an applied static electric field. In Sect. 2, we present some comments on the calculation scheme, followed by the discussion of the obtained results in Sect. 3. Finally, some conclusions are given in Sect. 4 .

\section{A brief outlook on the theoretical model}

The details of the solution for the electronic states in a system consisting of a quantum disc plus parabolic, inverse square and magnetic field potentials can be found in Ref. [3]. The effective mass and parabolic band approximations are the starting point for the calculation. The inclusion of an electric field oriented along $x=r \cos \theta$ (the Cartesian $x$-direction) prevents from the analytical solving of the two-dimensional effective mass conduction band Schrödinger problem. Therefore, we have used an approximate method based on the expansion of the electron states in terms of the complete set of eigenfunctions of the problem without electric field [4]. Having them in analytical form greatly simplifies the approach. Then, the diagonalization of the resulting Hamiltonian matrix leads to the desired band states.

The zero electric field case shows these states labeled by two quantum numbers. The number $l=0,1,2,3, \ldots$, and the magnetic number $m=0, \pm 1, \pm 2, \pm 3, \ldots$ At zero magnetic field, the true ground state is the $l=0, m=0$ one. But when there is a finite magnetic field applied, the actual ground state corresponds to a different combination of these two quantum numbers, much resembling the Aharonov-Bohm situation [5]. To ensure the suitable convergence of the calculation procedure for nonzero electric field, the orthogonal system of functions are those corresponding to the combinations of quantum numbers $\{n=0, \ldots 10 ; m=0, \pm 1, \ldots \pm 10\}$, which entails a total of 231 terms in the expansion.

\section{Results and discussion}

Our study considers a GaAs-based structure in which the two-dimensional carrier density is $\sigma=10^{13} \mathrm{~cm}^{-2}$. The input for the field intensities includes $F=10,20$, $30 \mathrm{kV} / \mathrm{cm}$, and $B=10,15,20 \mathrm{~T}$. The spectrum of electron levels (which we are going to label by the quantum number $l$ ) is very dense. Between the ground and the first excited states there are energy differences below $20 \mathrm{meV}$, in all cases. This means that we need to make a proper 
selection of the intersubband transitions in order to report rather measurable quantities. On the other hand, we must restrict to include in the analysis only a number of the lower allowed energy levels, which would be those actually occupied at low temperatures. In this sense, for the situation of the intersubband-related NOR, the decision has been to pick up transitions related with an energy difference of around $40-60 \mathrm{meV}$ that, at the same time, exhibit the larger possible intersubband dipole moment matrix element $M_{i j}=\left\langle\psi_{i}|r \cos \theta| \psi_{j}\right\rangle$. Accordingly, the NOR coefficients depicted in Figs. 1 to 3 correspond to $l_{i}=1$ and $l_{j}=9(F=20 \mathrm{kV} / \mathrm{cm} ; B=10 \mathrm{~T}) ; l_{i}=1$ and $l_{j}=8(F=30 \mathrm{kV} / \mathrm{cm} ; B=15 \mathrm{~T})$, and $l_{i}=1$ and $l_{j}=8(F=40 \mathrm{kV} / \mathrm{cm} ; B=20 \mathrm{~T})$ cases, respectively.
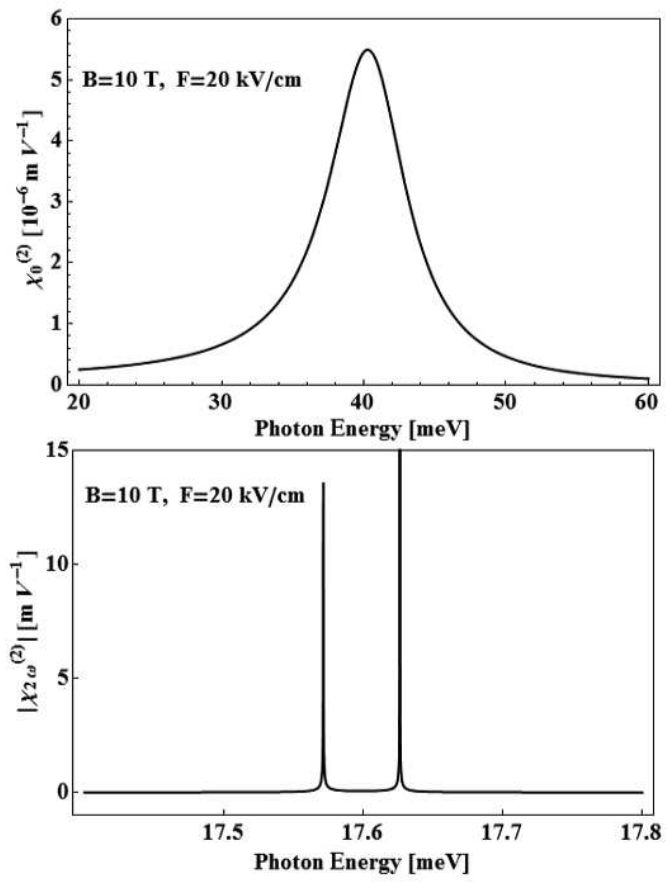

Fig. 1. Nonlinear optical rectification coefficient (top) and second harmonic generation coefficient (bottom) in a GaAs quantum ring under the combined influences of an electric and a magnetic field, as functions of the incident photon energy. The results correspond to the situation of an electric field of intensity $F=20 \mathrm{kV} / \mathrm{cm}$ and a static magnetic field strength $B=10 \mathrm{~T}$.

It can be seen a blueshift of the resonant NOR peak (notice that the second and third cases correspond to the same transition), together with a reduction in its amplitude. The increase in the field intensities causes the increment in the energy distance between the levels (for $F=0$, it readily follows from the corresponding exact analytical expression - see Eq. (16) of Ref. [3]), which reflects in the shift towards higher frequencies.

On the other hand, the fall in the NOR resonant peak amplitude relates mostly with the effect of the externally applied electric field. It causes a redistribution of the electron wave function in the region of the $\mathrm{QR}$, looking
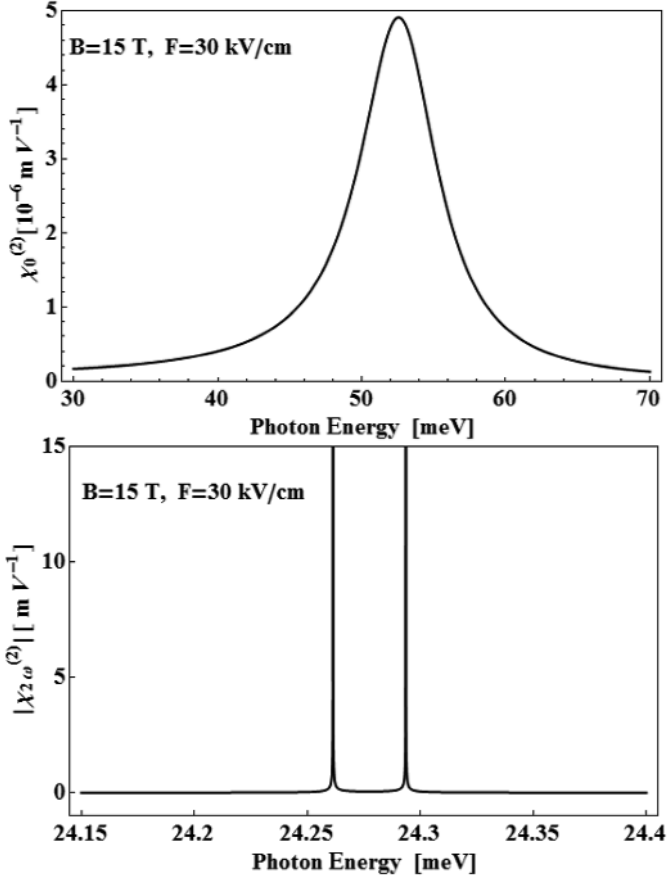

Fig. 2. Nonlinear optical rectification coefficient (top) and second harmonic generation coefficient (bottom) in a GaAs quantum ring under the combined influences of an electric and a magnetic field, as functions of the incident photon energy. The results correspond to the situation of an electric field of intensity $F=30 \mathrm{kV} / \mathrm{cm}$ and a static magnetic field strength $B=15 \mathrm{~T}$.

to preserve the orthogonality between states of different $l$. As a result of this, the value of integrand $M_{i j}$ in the region reduces thus leading to the decrease in the intersubband dipole moment matrix element.

Regarding the SHG coefficient, the task has been to detect electron transitions with large enough electric dipole moment matrix elements. That would lead to obtaining the highest possible second order harmonics generation. Such a procedure implies selecting a suitable three-level transition. The choice made is that with $l_{1}=2, l_{2}=5$, and $l_{3}=10$, as initial, middle, and upper level, respectively, for the three different configurations of electric and magnetic field intensities reported. The features of the obtained electron spectra has allowed to detect this particular three-level transition, which almost exactly reproduces the ideal energy spacing $\left(E_{12}=\hbar \omega ; E_{23}=2 \hbar \omega\right)$ for the SHG, in the three situations under study. In this case, we can also observe the blueshift of the resonant peaks commented previously in the discussion of the NOR coefficient when the field intensities augment. The same arguments given above are useful to explain such a displacement.

The magnitudes of the SHG coefficients reveal significantly high. This allows us to identify our GaAs QR nanosystem with external magnetic and electric fields as a possible source of frequency-doubling in the far infrared. 

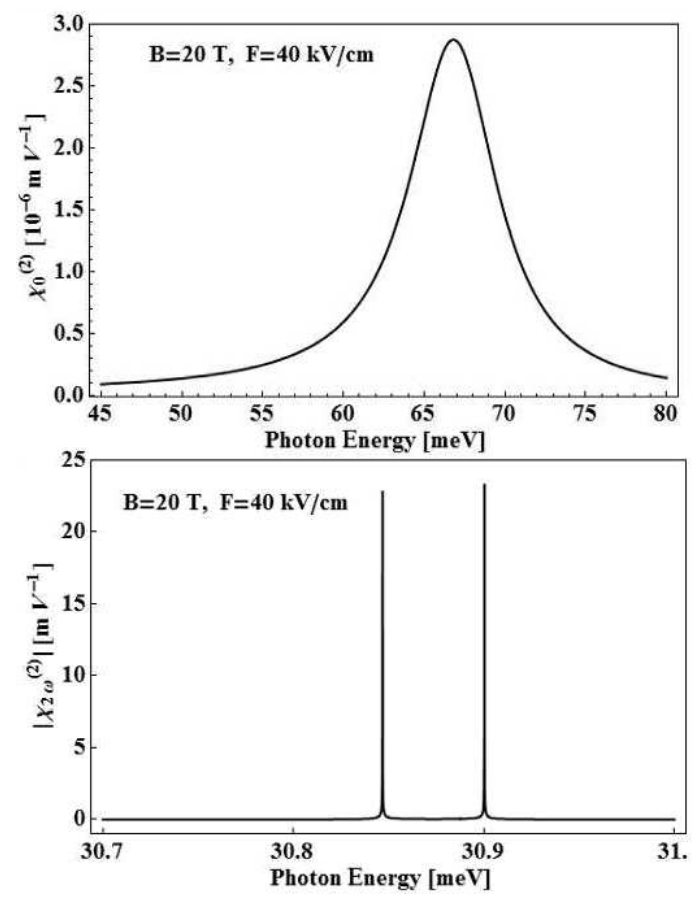

Fig. 3. Nonlinear optical rectification coefficient (top) and second harmonic generation coefficient (bottom) in a GaAs quantum ring under the combined influences of an electric and a magnetic field, as functions of the incident photon energy. The results correspond to the situation of an electric field of intensity $F=40 \mathrm{kV} / \mathrm{cm}$ and a static magnetic field strength $B=20 \mathrm{~T}$.

\section{Conclusions}

In this work we have studied the second order optical response of GaAs quantum rings, modeled from considering a two-dimensional quantum disc plus inverse square and parabolic confining potentials, and including the effect of externally applied electric and magnetic fields. The calculated nonlinear optical rectification and second harmonic generation coefficients show resonant peak blueshifts as long as the external field intensities increase. On the other hand, it is possible to identify specific three-level energy transitions in this kind of systems for which the magnitude of the second harmonic generation coef- ficient can be significantly high. This result points to a plausible application of the structures studied as source of higher harmonics generation in the infrared range.

\section{Acknowledgments}

M.E.M.R. acknowledges support from Mexican CONACYT through grant CB-2008-101777, and through sabbatical grant 2011-2012 No. 18036. He also thanks Universidad de Antioquia for hospitality during his sabbatical stay. This research was partially supported by Colombian Agencies: CODI-Universidad de Antioquia (Estrategia de Sostenibilidad 2013-2014 de la Universidad de Antioquia and the project: E01535-Efectos de la presión hidrostática y de los campos eléctrico y magnético sobre las propiedades ópticas no lineales de puntos, hilos y anillos cuánticos de GaAs-(Ga,Al)As y Si $\left./ \mathrm{SiO}_{2}\right)$, Facultad de Ciencias Exactas y Naturales-Universidad de Antioquia (C.A.D. - exclusive dedication project 2012-2013), and by el Patrimonio Autónomo Fondo Nacional de Financiamiento para la Ciencia, la Tecnología y la Innovacion, Francisco José de Caldas. M.E.M.R. and C.A.D. thank CONACYT (México) and COLCIENCIAS (Colombia) for support under the 2012-2013 Bilateral Agreement "Estudio de propiedades ópticas, electrónicas y de transporte en sistemas de baja dimensión basados en carbono y semiconductores III-V: efectos de campos externos, temperatura y presión hidrostática". The work was developed with the help of CENAPAD-SP, Brazil.

\section{References}

[1] E. Rosencher, Ph. Bois, Phys. Rev. B 44, 11315 (1991).

[2] G. Liu, K. Guo, C. Wang, Physica B 407, 2334 (2012).

[3] C.M. Duque, M.E. Mora-Ramos, C.A. Duque, Ann. Phys. 524, 327 (2012).

[4] J.M. Llorens, C. Trallero-Giner, A. García-Cristobal, A. Cantarero, Phys. Rev. B 64, 035309 (2001).

[5] B. Grbic, R. Leturcq, T. Ihn, K. Ensslin, D. Reuter, A.D. Wieck, Physica E 40, 1273 (2008). 